2007 110: 3744-3752

Prepublished online August 22, 2007;

doi:10.1182/blood-2007-05-093294

\title{
MLN3897, a novel CCR1 inhibitor, impairs osteoclastogenesis and inhibits the interaction of multiple myeloma cells and osteoclasts
}

Sonia Vallet, Noopur Raje, Kenji Ishitsuka, Teru Hideshima, Klaus Podar, Shweta Chhetri, Samantha Pozzi, Iris Breitkreutz, Tanyel Kiziltepe, Hiroshi Yasui, Enrique M. Ocio, Norihiko Shiraishi, Janice Jin, Yutaka Okawa, Hiroshi Ikeda, Siddhartha Mukherjee, Nileshwari Vaghela, Diana Cirstea, Marco Ladetto, Mario Boccadoro and Kenneth C. Anderson

Updated information and services can be found at:

http://bloodjournal.hematologylibrary.org/content/110/10/3744.full.html

Articles on similar topics can be found in the following Blood collections

Chemokines, Cytokines, and Interleukins (564 articles)

Hematopoiesis and Stem Cells (2985 articles)

Neoplasia (4217 articles)

Information about reproducing this article in parts or in its entirety may be found online at:

http://bloodjournal.hematologylibrary.org/site/misc/rights.xhtml\#repub_requests

Information about ordering reprints may be found online at:

http://bloodjournal.hematologylibrary.org/site/misc/rights.xhtml\#reprints

Information about subscriptions and ASH membership may be found online at:

http://bloodjournal.hematologylibrary.org/site/subscriptions/index.xhtml

Blood (print ISSN 0006-4971, online ISSN 1528-0020), is published weekly by the American Society of Hematology, 2021 L St, NW, Suite 900,

Washington DC 20036.

Copyright 2011 by The American Society of Hematology; all rights reserved.

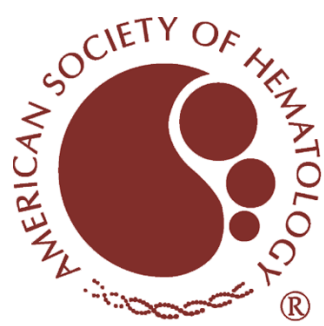




\title{
MLN3897, a novel CCR1 inhibitor, impairs osteoclastogenesis and inhibits the interaction of multiple myeloma cells and osteoclasts
}

\author{
Sonia Vallet, ${ }^{1,2}$ Noopur Raje, ${ }^{1,3}$ Kenji Ishitsuka, ${ }^{1}$ Teru Hideshima, ${ }^{1}$ Klaus Podar, ${ }^{1}$ Shweta Chhetri, ${ }^{1}$ Samantha Pozzi, ${ }^{1}$ \\ Iris Breitkreutz, ${ }^{1}$ Tanyel Kiziltepe, ${ }^{1}$ Hiroshi Yasui, ${ }^{1}$ Enrique M. Ocio, ${ }^{1}$ Norihiko Shiraishi, ${ }^{1}$ Janice Jin, ${ }^{1}$ Yutaka Okawa, ${ }^{1}$ \\ Hiroshi Ikeda, ${ }^{1}$ Siddhartha Mukherjee, ${ }^{3}$ Nileshwari Vaghela, ${ }^{1}$ Diana Cirstea, ${ }^{1}$ Marco Ladetto, ${ }^{2}$ Mario Boccadoro, ${ }^{2}$ and \\ Kenneth C. Anderson 1 \\ 1Jerome Lipper Multiple Myeloma Disease Center, Dana-Farber Cancer Institute, Harvard Medical School, Boston, MA; 2 Division of Hematology, University of \\ Turin, Turin, Italy; and ${ }^{3}$ Division of Hematology and Oncology, Massachusetts General Hospital, Boston
}

\begin{abstract}
The interaction between osteoclasts (OCs) and multiple myeloma (MM) cells plays a key role in the pathogenesis of MMrelated osteolytic bone disease (OBD). $M M$ cells promote OC formation and, in turn, OCs enhance MM cell proliferation. Chemokines are mediators of MM effects on bone and vice versa; in particular, CCL3 enhances OC formation and promotes MM cell migration and survival. Here, we characterize the effects of MLN3897, a novel specific antagonist of
\end{abstract}

the chemokine receptor CCR1, on both OC formation and OC-MM cell interactions. MLN3897 demonstrates significant impairment of OC formation (by $40 \%$ ) and function (by $70 \%$ ), associated with decreased precursor cell multinucleation and down-regulation of c-fos signaling. OCs secrete high levels of CCL3, which triggers MM cell migration; conversely, MLN3897 abrogates its effects by inhibiting Akt signaling. Moreover, MM cellto-OC adhesion was abrogated by
MLN3897, thereby inhibiting MM cell survival and proliferation. Our results therefore show novel biologic sequelae of CCL3 and its inhibition in both osteoclastogenesis and MM cell growth, providing the preclinical rationale for clinical trials of MLN3897 to treat OBD in MM. (Blood. 2007;110:3744-3752)

๑) 2007 by The American Society of Hematology

\section{Introduction}

Osteolytic bone disease (OBD), characterized by bone pain, increased risk of fracture, and tumor-induced hypercalcemia, is a frequent complication of multiple myeloma (MM). The yearly incidence of skeletal-related events in patients with $\mathrm{MM}$ is approximately $40 \%$; in particular, vertebral fractures account for $12 \%$ to $30 \%$, with significant clinical and economic impact. ${ }^{1}$ OBD is due to an imbalance between bone resorption and bone formation. Malignant plasma cells secrete osteoclast (OC) activating factors, such as interleukin-6 (IL-6), receptor activator of nuclear factor- $\mathrm{B}$ ligand (RANKL), and CCL3, which induce osteoclastogenesis and stimulate $\mathrm{OC}$ activity. Moreover, they also inhibit osteoblast (OB) formation via production of DKK1, thereby up-regulating OCs. Conversely, OCs support MM cell survival, inducing tumor cell growth and chemotherapy resistance. ${ }^{2}$ Therefore, new agents targeting this MM-OC interaction are needed.

Chemokines are chemotactic cytokines involved in regulating the immune system, mainly by directing the homing of circulating leukocytes to sites of inflammation. ${ }^{3}$ They bind $G$ protein-coupled receptors, thereby stimulating several signaling pathways. ${ }^{3,4}$ Chemokines contribute to the pathogenesis of a variety of diseases ${ }^{3,5}$; in particular, CCL3 plays a key role in MM. It stimulates migration and proliferation of MM cells by activation of Akt and mitogen-activated protein kinase (MAPK) signaling pathways, ${ }^{6}$ and promotes OC formation independently

Submitted May 31, 2007; accepted August 10, 2007. Prepublished online as Blood First Edition paper, August 21, 2007; DOI 10.1182/blood-200705-093294.

The online version of this article contains a data supplement. of RANKL. ${ }^{7}$ Importantly, targeting CCL3 with neutralizing antibodies or siRNA directly reduces bone lesions and tumor burden in MM mouse models. ${ }^{8,9}$ MM cells express both receptors for CCL3, CCR1, and CCR5. ${ }^{6,10}$ Moreover, CCR1 is the major receptor on OC precursor cells and mature OCs ${ }^{11,12}$; therefore, CCR1 is a promising novel therapeutic target for OBD in MM.

In this study, we characterized the effects of a specific CCR1 inhibitor MLN3897 (Millennium Pharmaceuticals, Cambridge, MA) on formation and function of OCs, as well as on the interactions between OCs and MM cells. MLN3897 is an oral clinical-grade small-molecule receptor antagonist currently under evaluation in phase 2 clinical trials for rheumatoid arthritis and multiple sclerosis. Here, we show that MLN3897 inhibits osteoclastogenesis and OC activity by impairing multinucleation and by down-regulation of c-fos signaling. It also abrogates CCL3-induced MM cell migration by Akt inhibition. Furthermore, MLN3897 inhibits OC-stimulated MM cell migration and adhesion, and abrogates the protective effects of OCs on MM cell survival and proliferation. Taken together, these data identify a novel mechanism of action for MLN3897 against osteoclastogenesis and OC-mediated MM survival. These studies therefore provide the preclinical rationale for the clinical evaluation of MLN3897 to treat patients with MM-related OBD. 


\section{Patients, materials, and methods}

\section{MLN3897}

MLN3897, an orally active, small-molecule-specific antagonist of CCR1, was obtained from Millennium Pharmaceuticals. It has an $\mathrm{IC}_{50}$ of $0.8 \mathrm{nM}$ for CCR 1 and $4 \mu \mathrm{M}$ for CCR5, determined by competition binding assays of fluorescently labeled CCL3 to CCR1- and CCR5-expressing cells. It is highly selective for human CCR1 and does not inhibit the effects of CCL3 on mouse leukocytes. Moreover, it does not inhibit RAF, Akt, or receptor tyrosine kinases at concentrations up to $10 \mu \mathrm{M}$. MLN3897 was dissolved in dimethyl sulfoxide (DMSO; Sigma Chemical, St Louis, MO) at $10 \mathrm{mM}$, and stored at $-20^{\circ} \mathrm{C}$ until use. For each experiment, it was diluted immediately before use in culture medium $(0.2-100 \mathrm{nM})$ with less than $0.002 \%$ of DMSO. Since MLN3897 is a highly stable compound with a half-life of 3 days, it was added twice weekly to culture medium.

\section{Cell lines and primary MM cells}

Cell lines. The dexamethasone (Dex)-sensitive (MM.1S) human MM cell line was kindly provided by Dr Steven Rosen (Northwestern University, Chicago, IL). The INA6 human IL-6-dependent MM cell line was provided by Dr Renate Burger ${ }^{13}$ (University of Kiel, Kiel, Germany) and cultured in the presence of $2.5 \mathrm{ng} / \mathrm{mL}$ IL-6 (R\&D Systems, Minneapolis, MN). The OPM1 myeloma cell line was kindly provided by Dr Lief Bergsagel (Mayo Clinic, Scottsdale, AZ), and the U266 cell line was obtained from the American Type Culture Collection (Rockville, MD). All MM cell lines were cultured in RPMI 1640 media (Sigma Chemical) containing 10\% fetal bovine serum (FBS), $2 \mathrm{mM}$ L-glutamine, $100 \mathrm{U} / \mathrm{mL}$ penicillin, and 100 $\mu \mathrm{g} / \mathrm{mL}$ streptomycin (Gibco, Grand Island, NY).

MM primary cells. Patient tumor cells were isolated as previously described. ${ }^{14}$ Briefly, after appropriate informed consent was obtained in accordance with the Declaration of Helsinki and with approval by the Institutional Review Board of the Dana-Farber Cancer Institute (Boston, MA), MM patient cells were separated from bone marrow (BM) samples by antibody-mediated positive selection using anti-CD138 magnetic activated cell separation microbeads (Miltenyi Biotech, Gladbach, Germany).

\section{$\mathrm{OC}$ formation and activity assays}

OC formation and TRAP assay. OCs were generated from peripheral blood mononuclear cells (PBMCs) from healthy volunteers by Ficoll-Paque gradient separation and cultured in 6-well or 96-well plates $\left(0.5 \times 10^{6}\right.$ cells $/ \mathrm{cm}^{2}$ ). After 2 hours, nonadherent PBMCs were removed, and adherent cells were cultured for 21 days in $\alpha$-MEM containing $10 \%$ FBS and $1 \%$ penicillin-streptomycin (Mediatech, Herndon, VA), as well as $50 \mathrm{ng} / \mathrm{mL}$ of macrophage colony-stimulating factor (M-CSF; R\&D Systems, Minneapolis, MN) and RANKL (PeproTech, Rocky Hill, NJ). MLN3897 was added at concentrations and time points as indicated; culture media was replaced twice weekly. After 3 weeks, cells were fixed with citrate-acetone solution and stained for tartrate-resistant acid phosphatase (TRAP) using an acid phosphatase leukocyte staining kit (Sigma Chemical) according to the

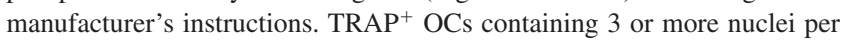
cell were enumerated. Each OC formation assay was performed at least 3 times using PBMCs from different donors.

Pit formation assay. OC activity was assayed by bone resorption enumerating resorption pits. Briefly, PBMCs were cultured $\left(0.5 \times 10^{6}\right.$ cells/well) on dentin slices (Immunodiagnostic Systems, Boldon, United Kingdom) in 96-well plates as per the manufacturer's guidelines, and then stimulated with RANKL and M-CSF (50 ng/mL); MLN3897 was added as indicated. After 3 weeks, adherent cells were scraped off gently with $0.1 \%$ Triton. Bone slices were washed in distilled water and stained with $1 \%$ toluidine solution. Resorption pits were then quantified by light microscopy using the public domain National Institutes of Health (NIH) Image J software version $1.36 \mathrm{~b} .{ }^{15}$ Each pit area assay was performed at least 3 times with PBMCs from different donors.

Coculture experiments. OCs were harvested with cell dissociation buffer (Invitrogen, Carlsbad, CA) and seeded in 96-well or 24-well plates (approximately $1.5-3 \times 10^{4}$ cells $/ \mathrm{cm}^{2}$ ). After washing, MM cells were added to the wells and incubated with media or with MLN3897 (10 nM) for the specified times at $37^{\circ} \mathrm{C}$. For MM cell proliferation, we measured DNA synthesis by tritiated thymidine uptake $(3 \mathrm{H}-\mathrm{TdR}$; Perkin Elmer, Boston, MA), pulsing MM cells with $3 \mathrm{H}-\mathrm{TdR}(0.5 \mu \mathrm{Ci} /$ well $[0.0185 \mathrm{MBq}] \mathrm{L})$ during the last 8 hours of 48 -hour cultures. At the end of the culture, cells were harvested onto paper filters with an automatic cell harvester (Cambridge Technology, Cambridge, MA) and counted using the LKB Betaplate scintillation counter (Wallac, Gaithersburg, MD). To assess cell survival, viable MM cells were counted by trypan blue staining.

\section{Cell viability and cell fusion assays of OC precursors}

We determined the effects of MLN3897 on viability and fusion of PBMCs stimulated with RANKL and M-CSF. At different time points, cell number was quantified using the AlamarBlue assay (BioSource International, Camarillo, CA), pulsing the cells with AlamarBlue $(10 \mu \mathrm{L})$ and incubating for 4 hours at $37^{\circ} \mathrm{C}$. Absorbance was read at a wavelength of $570 \mathrm{~nm}$ (with correction at $600 \mathrm{~nm}$ ) on a spectrophotometer (Molecular Devices, Sunnyvale, CA). To analyze cell fusion, cells were fixed in $3.7 \%$ formaldehyde for 5 minutes and then stained with hematoxylin and eosin (Sigma Chemical) for 5 and 10 minutes, respectively. Cell fusion, evidenced by cells with 3 or more nuclei, was quantified using light microscopy. Each fusion assay was performed at least 3 times with PBMCs from different donors.

\section{Analysis of cell-surface marker expression}

For analysis of cell-surface marker expression, cells were suspended in Dulbecco phosphate-buffered saline. Following 30 minutes incubation at $4^{\circ} \mathrm{C}$ with the primary antibody, cells were washed and analyzed using the RXP cytomics software on an Epics flow cytometer (Coulter Immunology, Hialeah, FL). We used affinity-purified monoclonal antibodies specific for CCR1 (phycoerythrin [PE]-conjugated mouse immunoglobulin IgG2a; R\&D Systems), CCR5 (PE-conjugated mouse immunoglobulin IgG2b; R\&D Systems), CD14 (PE-CyChrome 5-conjugated mouse immunoglobulin IgG2a; Beckman Coulter, Fullerton, CA), and VLA5 (CD49e or $\alpha \mathrm{V}$-integrin, fluorescein isothiocyanate [FITC]-conjugated monoclonal antibody; Beckman Coulter).

\section{ELISA}

CCL3, vascular endothelial growth factor (VEGF), CCL5, and IL-6 expression were analyzed by enzyme-linked immunosorbent assay (ELISA; R\&D Systems). We tested supernatants of MM cell lines, primary MM cells, monocytes, and mature OCs seeded in RPMI 1640 or $\alpha$-MEM with $10 \%$ FBS for 48 hours at a density of $10^{6}$ cells $/ \mathrm{mL}$. IL-6 secretion was also assessed in 48-hour supernatants of the cocultures between OCs and MM cell lines. All measurements were done in triplicate.

\section{Transwell migration assay}

Cell migration was assayed using a Boyden-modified chamber assay. Cells were added on an $8-\mu \mathrm{m}$ pore size polycarbonate membrane separating the 2 chambers of a $6.5-\mathrm{mm}$ Transwell (Corning, Corning, NY). CCL3 $(5 \mathrm{ng} / \mathrm{mL})$ or 48 -hour OC supernatants were added in the lower chamber. After 5 hours, cells migrating to the lower compartment were counted using a Coulter Counter ZBII (Beckman Coulter). In specific experiments, cells were preincubated with MLN3897 (10 nM, 4 hours) and specific PI3K inhibitor LY294002 (25 $\mu \mathrm{M}, 1$ hour).

\section{Western blotting}

Overnight serum-starved cells were stimulated with CCL3 $(100 \mathrm{ng} / \mathrm{mL}$ for 30 minutes) with or without pretreatment of MLN3897 (10 nM), harvested, and lysed in lysis buffer $\left(50 \mathrm{mM} \mathrm{N}\right.$-2-hydroxyethylpiperazine- $\mathrm{N}^{\prime}$-2ethanesulfonic acid [pH 7.4], $150 \mathrm{mM} \mathrm{NaCl}, 1 \% \mathrm{NP}-40,30 \mathrm{mM}$ sodium pyrophosphate, $5 \mathrm{mM}$ ethylenediamine tetraacetic acid, $2 \mathrm{mM} \mathrm{Na} 3 \mathrm{VO} 4$, $5 \mathrm{mM}$ sodium fluoride, $1 \mathrm{mM}$ phenylmethylsulfonyl fluoride, $5 \mu \mathrm{g} / \mathrm{mL}$ leupeptin, and $5 \mu \mathrm{g} / \mathrm{mL}$ aprotinin). For OC signaling experiments, adherent PBMCs were seeded in 6-well plates $\left(0.2 \times 10^{6} \mathrm{cells} / \mathrm{cm}^{2}\right)$, harvested with 
A
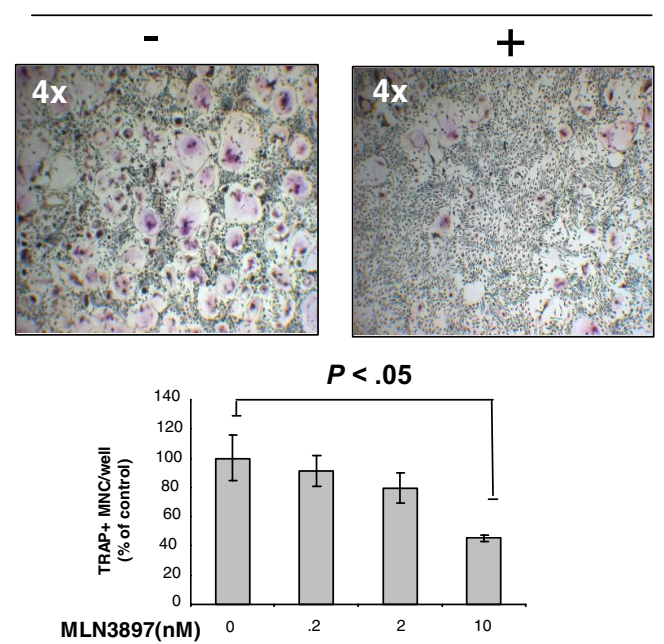

B
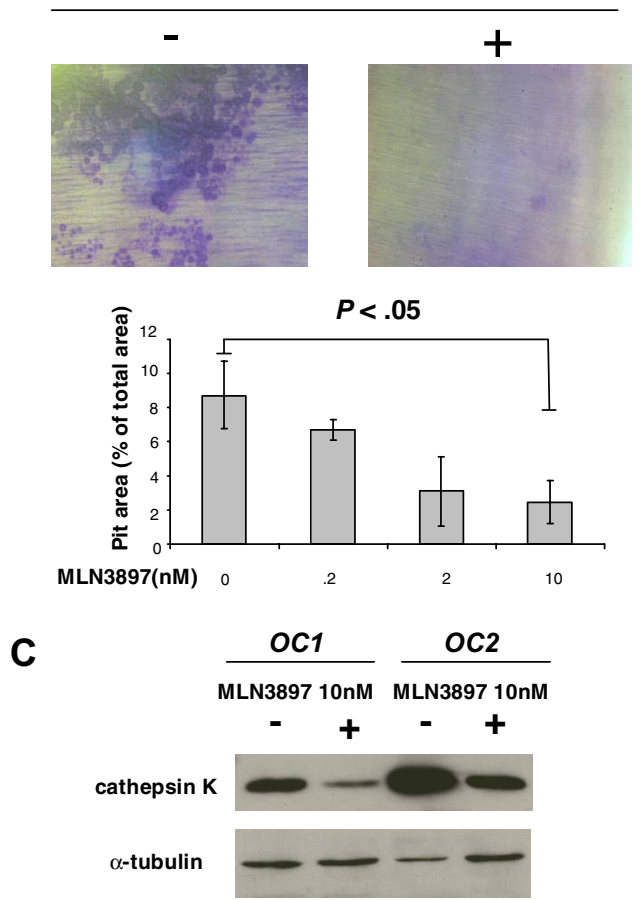

Figure 1. MLN3897 inhibits OC formation and function. Adherent PBMCs were cultured for 3 weeks in the presence of RANKL and M-CSF $(50 \mathrm{ng} / \mathrm{mL})$ with or without MLN3897 (0.2-10 nM). (A) Cells were stained for TRAP activity. Multinuclear $\mathrm{TRAP}^{+}$cells are expressed as percentage of control. (B) Adherent PBMCs were cultured on dentine slices. After 3 weeks, resorption areas were stained with toluidine blue, and pit areas were quantified by light microscopy using the public domain NIH Image J program. Each value represents the mean $( \pm S D)$ of resorptive areas of at least 3 wells, expressed as a percentage of total area. Images were obtained using a Leica DM IL microscope equipped with a $4 \times, 10 \times / 0.22$, and $20 \times / 0.40$ numeric aperture objective lens (Leica Microsystems, Wetzlar, Germany) and acquired through IM50 software (Leica Microsystems Imaging Solutions, Cambridge, United Kingdom). All experiments were performed independently at least 3 times. (C) After 3 weeks, we investigated cathepsin K expression on OCs obtained from 2 different donors and treated with MLN3897 (10 nM). Cells were harvested with cell dissociation buffer and lysed; proteins were subjected to immunoblotting with anticathepsin $\mathrm{K}$ antibody. To ensure equal protein loading, membrane was blotted for tubulin expression.

cell dissociation buffer (Invitrogen) after 7 days of stimulation with RANKL and M-CSF with or without MLN3897, and lysed in lysis buffer. Samples were then subjected to sodium dodecyl sulfate-polyacrylamide gel electrophoresis, transferred to PVDF membrane, and immunoblotted with antibodies against pAkt, Akt, and c-fos (Cell Signaling Technology, Beverly, MA), phosphorylated extracellular signal-regulated kinase (pERK), ERK (Santa Cruz Biotechnology, Santa Cruz, CA), or cathepsin K (Chemicon International, Temecula, CA). Antigen-antibody complexes were detected by enhanced chemiluminescence (Amersham, Arlington Heights, IL). The membrane was stripped and reprobed with antitubulin antibody to ensure equal protein loading. Films were scanned and densitometric analysis performed using the public domain NIH Image $\mathrm{J}$ program. ${ }^{15}$

\section{Adhesion assay}

To perform adhesion assays, MM cell lines were labeled with calcein AM (Invitrogen) according to the manufacturer's instructions and plated in a 96-well plates with OCs, fibronectin $(20 \mu \mathrm{g} / \mathrm{mL})$, or media, with or without MLN3897. After 6 hours of incubation, plates were washed and fluorescence of the adherent cells was measured using the Multimode Reader Mithras LB 940 (Berthold Technologies, Wildbad, Germany).

\section{Statistical analysis}

Each experiment was repeated at least 3 times, and all quantitative data are presented as means plus or minus SD. Statistical comparisons by the Student 2-tailed $t$ test were considered statistically significant at a $P$ level less than .05 .

\section{Results}

\section{MLN3897 inhibits OC formation and activity}

Since CCR 1 is expressed on the surface of OC precursors and promotes osteoclastogenesis, ${ }^{11,12}$ we first investigated whether MLN3897 inhibited OC formation and function. Adherent PBMCs from healthy donors were cultured in the presence of RANKL and M-CSF (50 ng/mL) for 21 days, with or without MLN3897. TRAP staining was then performed to enumerate multinucleated TRAP $^{+}$ cells. MLN3897 (10 nM) reduced OC number by $40 \%$ to $60 \%$ compared with control (Figure 1A; $P<.05$ ). No dose-dependent effect was noted, as doses up to $100 \mathrm{nM}$ did not further decrease OC number; therefore, all subsequent experiments used a dose of $10 \mathrm{nM}$ MLN3897.

To determine whether inhibition of OC formation by MLN3897 was associated with decreased bone resorption, we performed pit formation assays. As shown in Figure 1B, almost complete abrogation of the characteristic resorptive tracks and a reduction of pit numbers was noted in the presence of MLN3897 (10 nM). Consistent with the reduction of OC formation, MLN3897 (10 nM) significantly reduced bone resorption areas (mean $\pm \mathrm{SD}$, $2.4 \% \pm 1.2 \%$ vs $8.7 \% \pm 1.9 \%$ of total area per slice in the control; $P<.01)$. Concordantly, cathepsin $\mathrm{K}$ expression was decreased after treatment with MLN3897 (Figure 1C), suggesting that MLN3897 inhibited differentiation of OC precursors induced by RANKL and M-CSF.

\section{MLN3897 decreases fusion of OC precursors and down-regulates c-fos expression}

In order to identify the mechanism by which MLN3897 blocks osteoclastogenesis, we next performed time-course experiments culturing PBMCs with RANKL and M-CSF in the presence of MLN3897 (10 nM). The number of $\mathrm{TRAP}^{+}$multinucleated cells was significantly reduced compared with control after exposure to MLN3897 during the second week of cultures $(72 \pm 18$ cells/well compared with $160 \pm 24$ cells/well, respectively; Figure 2A). Accordingly, adding MLN3897 in the second week 
A

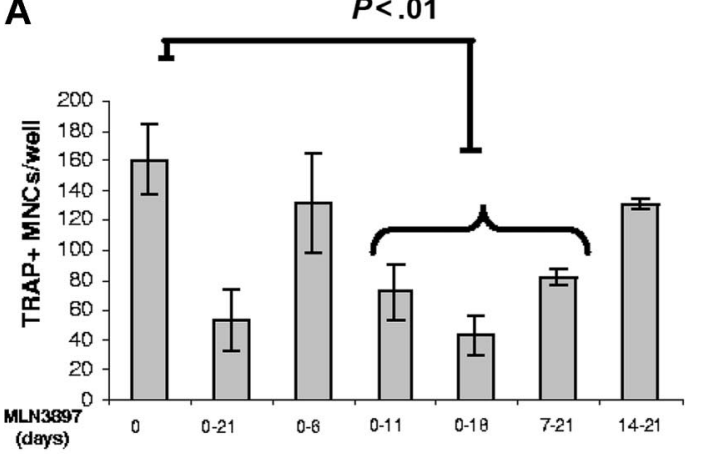

C

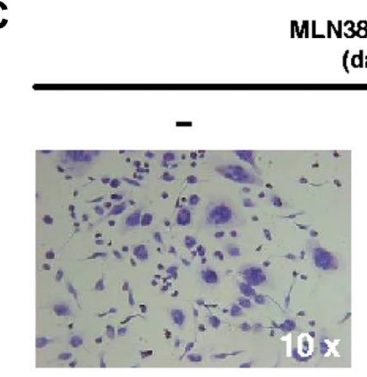

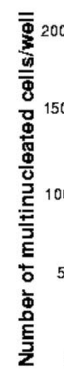

Days 0
MLN3897 $10 \mathrm{nM}$ (day 7)

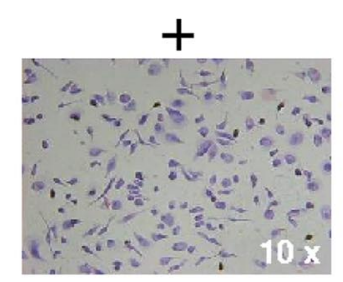

-๑- MLN3997 O NM

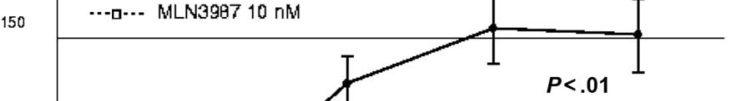

50

0
B

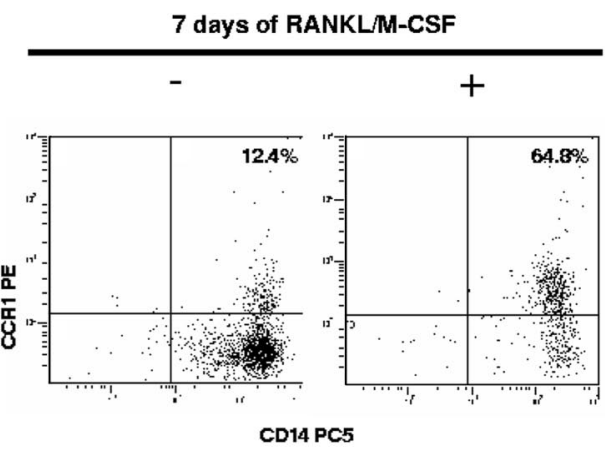

D

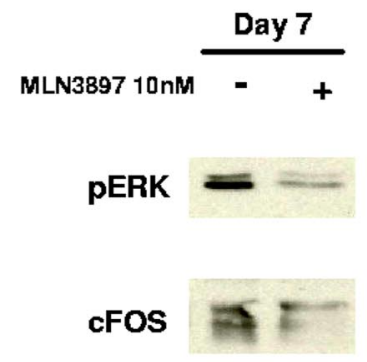

ERK

Figure 2. MLN3897 blocks fusion of OC precursor cells. (A) Adherent PBMCs cultured with RANKL and M-CSF (50 ng/mL) were exposed to MLN3897 (10 nM) for the indicated time points. After 3 weeks, TRAP ${ }^{+}$multinucleated cells were stained and counted. Results are expressed as number of TRAP ${ }^{+}$multinucleated cells per well: each value represents the mean ( \pm SD) of OCs per well of at least 3 wells. All experiments were performed independently at least 3 times. (B) CCR1 and CD14 coexpression was analyzed by flow cytometry on human PBMCs cultured with or without RANKL and M-CSF ( $50 \mathrm{ng} / \mathrm{mL})$ for 7 days. (C) PBMCs were cultured in the presence of RANKL and M-CSF with or without MLN3897 (10 nM). At the indicated time points, cells were fixed and stained with hematoxylin/eosin. Cells with more than 3 nuclei were enumerated; images were obtained with a light microscope (Leica Microsystems, Wetzlar, Germany) and acquired through IM50 software (Leica Microsystems Imaging Solutions, Cambridge, United Kingdom). (D) At day 7, PBMCs stimulated with RANKL and M-CSF (50 ng/mL) in the presence or absence of MLN3897 (10 nM) were harvested and lysed; proteins were immunoblotted with anti-pERK, ERK, and c-fos antibodies.

(days 7-14) of PBMC culture resulted in a significant impairment of OC generation (data not shown). These data suggest that MLN3897 interferes with OC differentiation, after commitment of precursor cells.

Importantly, CCR1 expression is up-regulated during differentiation of human monocytes to OCs triggered by RANKL and M-CSF. Compared with unstimulated PBMCs, OC precursors stimulated by RANKL and M-CSF for 7 days express CCR 1 at high levels (64\% vs 12\%; Figure 2B), confirming a role for CCR1 at this stage of osteoclastogenesis. Since chemokines mediate the fusion of OC progenitors into multinucleated $\mathrm{TRAP}^{+} \mathrm{OCs}^{16}{ }^{16}$ we next investigated the effects of MLN3897 on the fusion stage by quantifying multinucleated cells (MNCs) generated in the presence or absence of MLN3897. Consistent with our time-course TRAP assays (Figure 2A), MLN3897 inhibits fusion of OC precursors (Figure 2C); at 7 days, MLN3897 (10 nM) significantly reduced MNC number by $70 \%$ (mean $\pm \mathrm{SD}, 37 \pm 11 \mathrm{MNCs} /$ well) compared with control (mean $\pm \mathrm{SD}, 121 \pm 16 \mathrm{MNCs} /$ well; $P=.008)$. This inhibitory effect of MLN3897 on OC formation was not associated with cell death, analyzed by AlamarBlue assay (data not shown).
Among signaling pathways involved in OC differentiation, ERK signaling has been shown as a critical regulator of OC precursor cell fusion. ${ }^{17}$ Importantly, MLN3897 (10 nM) downregulated $\mathrm{pERK}$ and its downstream target $\mathrm{c}$-fos, ${ }^{18,19}$ which are implicated in cell multinucleation ${ }^{20}$ (Figure 2D). Our data therefore suggest that MLN3897 interferes with the fusion of OC precursors, associated with down-regulation of pERK and c-fos expression.

\section{MM cells have modest CCR1 expression and secrete CCL3}

Although the CCL3/CCR1 pathway mediates several effects in MM cells, including migration, ${ }^{10}$ adhesion, ${ }^{21}$ and proliferation, ${ }^{6}$ CCR1 expression on MM cell lines is still controversial. ${ }^{6,10,21}$ We therefore analyzed surface CCR 1 expression on several MM cell lines by flow cytometry (Figure 3A; Table 1). Overall CCR1 expression was modest: MM1.S cells had the highest expression level (mean fluorescence intensity $[\mathrm{MFI}] \pm \mathrm{SD}, 1.2 \pm 0.3 \mathrm{vs}$ $0.6 \pm 0.1$ of isotype control [IC]); OPM1 and MM1.R displayed MFI levels of 1.3 plus or minus 0.5 and 1.5 plus or minus 0.1 , respectively, whereas INA6, LR5, RPMI, and U266 did not 
Table 1. Expression on MM cell lines and MM patient cells

\begin{tabular}{llc}
\hline Cell line & IC, MFI $( \pm$ SD & CCR1, MFI ( \pm SD $)$ \\
\hline MM1.s & $0.6( \pm 0.1)$ & $1.17( \pm 0.3)$ \\
OPM1 & $0.71( \pm 0.2)$ & $1.26( \pm 0.5)$ \\
MM1.r & $1( \pm 0.1)$ & $1.46( \pm 0.14)$ \\
RPMI & $1.3( \pm 0.5)$ & $1.6( \pm 0.3)$ \\
LR5 & $0.48( \pm 0.2)$ & $0.56( \pm 0.3)$ \\
INA6 & $0.8( \pm 0.5)$ & $0.9( \pm 0.48)$ \\
U266 & $0.6( \pm 0.1)$ & $0.7( \pm 0.3)$ \\
Primary MM cells & $0.5( \pm 0.2)$ & $0.6( \pm 0.3)$ \\
Monocytes & $0.7( \pm 0.5)$ & $3.47( \pm 1)$ \\
\hline
\end{tabular}

express CCR1 (Figure 3A; Table 1). Consistent with these data, primary MM cells expressed low levels of CCR1 (MFI, $0.6 \pm 0.3$ compared with $0.5 \pm 0.2$ of IC; Figure $3 \mathrm{~A}$; Table 1 ). We also analyzed CCR5 expression on 4 cell lines: MM.1S and OPM1 had the highest CCR1 expression, whereas U266 and INA6 were both negative for CCR1 expression. MM1.S cells expressed CCR5 at a lower intensity than CCR1 (MFI, $1 \pm 0.3$ vs IC $0.7 \pm 0.2$ ), INA6 cells showed modest CCR5 levels (MFI, $1 \pm 0.5$ vs IC $0.7 \pm 0.2)$, whereas OPM1 and U266 cell lines did not express CCR5 (Figure 3B).

Among CCR1 ligands, CCL3 plays a critical role in the pathogenesis of OBD in MM. ${ }^{7,22}$ Consistent with previous studies, ${ }^{6,23}$ we found that all MM cells secrete CCL3: the mean CCL3 level in culture supernatants of MM cell lines and MM primary cells was $1180 \mathrm{pg} / \mathrm{mL}$ (range, 28-4789 pg/mL) and $255 \mathrm{pg} / \mathrm{mL}$ (range, 35.5-581 pg/mL), respectively. MM1.S and U266 cell lines secreted high levels of CCL3 (mean \pm SD, $2.8 \pm 0.24$ and $4.8 \pm 0.17 \mathrm{ng} / \mathrm{mL}$, respectively), while INA6 and OPM1 cell lines secreted only $45( \pm 2.6)$ and $47( \pm 0.9) \mathrm{pg} / \mathrm{mL}$, respectively (Figure 3C).

\section{MLN3897 inhibits MM cell migration and adhesion}

CCL3 is a potent chemoattractant for MM cells, $6,10,24$ although the receptor mediating these effects is not defined. We therefore next investigated whether low doses $(5 \mathrm{ng} / \mathrm{mL})$ of CCL3 stimulated MM cell migration, and whether pretreatment with MLN3897 affected this response. Interestingly, migration of MM cell lines induced by
CCL3 correlated with the intensity of CCR1 expression. Migration was induced in MM1.S (1.9-fold) and OPM1 (1.2-fold) cells by CCL3; conversely, pretreatment with MLN3897 (10 nM for 4 hours) abrogated this response (Figure 4A). No migratory response to CCL3 was induced in $\mathrm{CCR}^{-}{ }^{-}$cell lines (Figure 4A). MM1.S cells expressed both receptors for CCL3, CCR1 (14\%), and CCR5 (4\%), whereas OPM1 cells expressed only CCR1 (Figure 3; Table 1), suggesting that CCL3 may bind to both receptors on MM1.S cells, thereby inducing a stronger migratory response than in OPM1 cells. Treatment with MLN3897 partially inhibited MM1.S migration by acting on CCR1, although effects on CCR5 cannot be excluded.

Since Akt is the major downstream molecule in CCL3mediated signaling cascade, we examined whether MLN3897 inhibits Akt phosphorylation triggered by CCL3. As expected, CCL3 (100 ng/mL for 30 minutes) induces Akt phosphorylation'; conversely, pretreatment with MLN3897 (10 nM for 4 hours) inhibited Akt phosphorylation partially in MM1S and completely in OPM1 cells (Figure 4B). These results also suggest that CCL3 may activate both receptors on MM.1S cells, and that MLN3897 mainly inhibited CCR1-mediated effects. In contrast, no Akt activation was observed in the CCR $1^{-}$cell lines INA6 and U266 (data not shown). Since the PI3K/Akt signaling pathway is involved in migration induced by several chemokines, ${ }^{25}$ we next determined whether CCL3 stimulated migration via PI3K/Akt signaling, using the PI3K inhibitor LY294002. Pretreatment with either MLN3897 (10 nM for 4 hours) or LY294002 (25 $\mu \mathrm{M}$ for 1 hour) significantly abrogated CCL3induced migration in both OPM1 and MM.1S cells (Figure 4C), suggesting that CCL3 stimulates migration via activating the PI3K/Akt pathway.

Mature OCs secrete several cytokines. ${ }^{2,26}$ In 48-hour OC culture supernatants, we identified CCL3 (mean, $1138.9 \mathrm{pg} / \mathrm{mL}$; range, 68-3768 pg/mL), IL-6 (mean, $159.4 \mathrm{pg} / \mathrm{mL}$; range, 11.3-326.5 $\mathrm{pg} / \mathrm{mL}$ ), VEGF (mean, $115.5 \mathrm{pg} / \mathrm{mL}$; range, 26-343 pg/mL), and CCL5 (mean, $20.6 \mathrm{pg} / \mathrm{mL}$; range, 12.3-27.5 pg/mL; Figure 5A). Consistent with these results, 48-hour OC culture supernatants stimulated MM.1S cell migration by 1.6-fold, whereas no stimulation was noted for INA6 (Figure 5B). Interestingly, pretreatment with MLN3897 (10 nM) partially inhibited MM1.S cell migration,
A

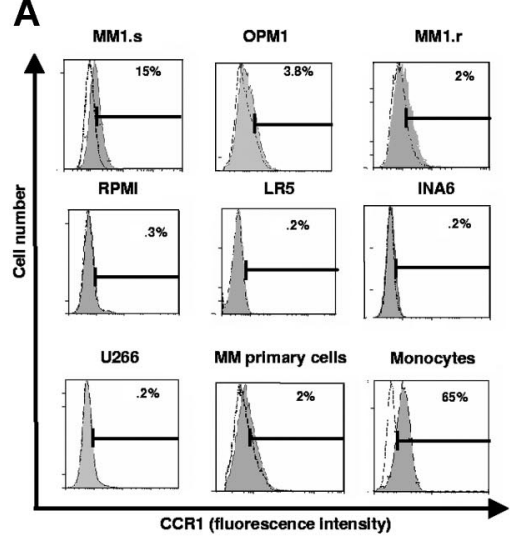

B

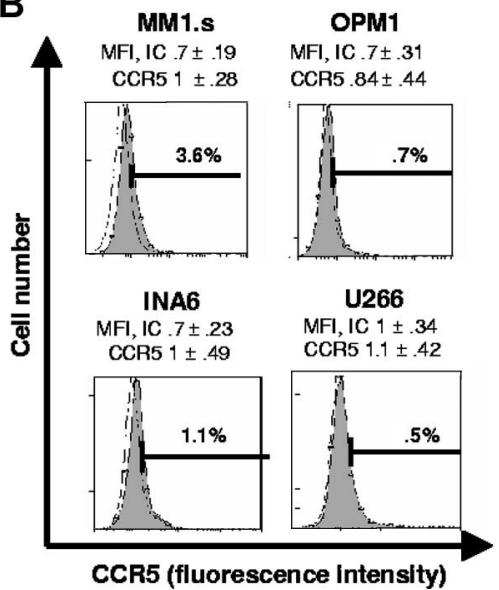

C

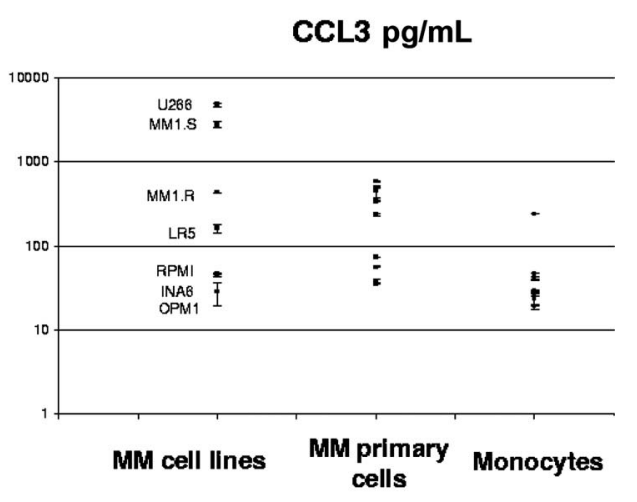

Figure 3. CCR1 expression and CCL3 secretion by MM cell lines and primary MM cells. (A) CCR1 expression on MM cell lines and patient MM cells was analyzed by flow cytometry. A representative flow cytometry shows percentage of positive cells from at least 3 different experiments. CCR1 expression on monocytes is shown as a positive control. (B) CCR5 expression on MM1.S, OPM1, INA6, and U266 was analyzed. Flow cytometry data represent several independent experiments. (C) CCL3 secretion was assessed by ELISA on 48-hour culture supernatants of MM cell lines, MM primary cells, and monocytes plated at a density of $10^{6}$ cells/mL. Means ( \pm SD) are expressed as $\mathrm{pg} / \mathrm{mL}$ on a logarithmic scale. 
A

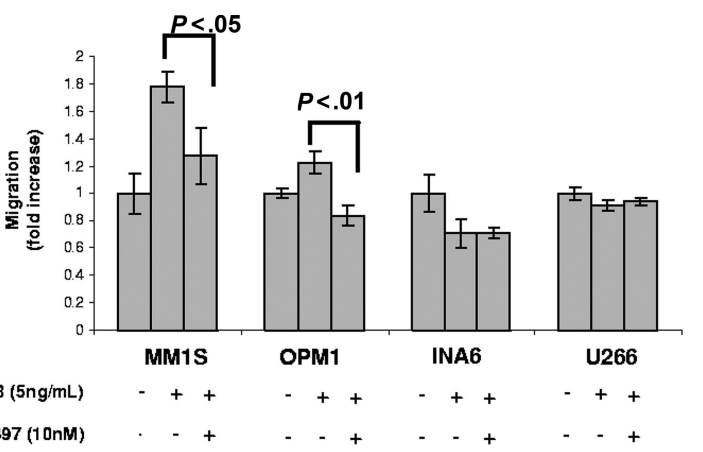

B

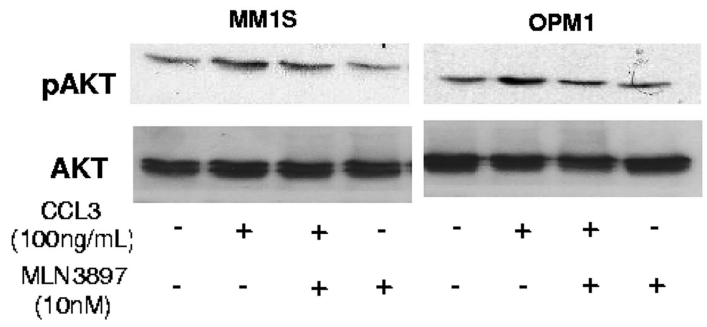

Relative protein expression (densitometric units)
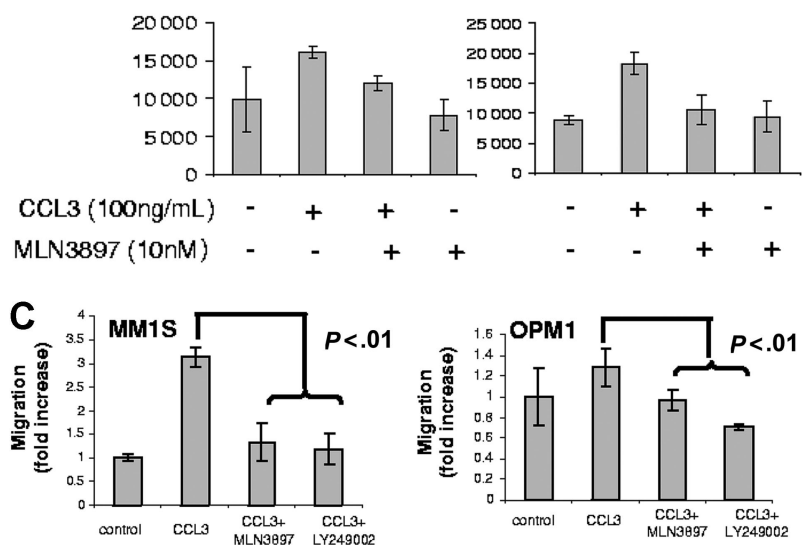

Figure 4. MLN3897 abrogates CCL3-induced migration by inhibiting Akt phosphorylation. Effects of MLN3897 on cell migration in the presence of CCL3 ( $5 \mathrm{ng} / \mathrm{mL}$ ) were assessed by Transwell migration assay. MM cells were preincubated with MLN3897 (10 nM for 4 hours) (A) or PI3K inhibitor LY94002 (25 $\mu \mathrm{M}$ for 1 hour) (C), and then seeded on the upper chamber of the Transwell plate. After 4 hours, cells that migrated to the lower chamber were counted, and results were expressed as fold increase over control plus or minus standard deviation. (B) CCL3 (100 ng/mL for 30 minutes) stimulated Akt phosphorylation in serum-starved (overnight in $1 \%$ and 30 minutes in $0 \%$ RPMI media) MM cells, assessed by Western blot analysis. In selected experiments, cells were preincubated with MLN3897 for 4 hours. The densitometric analysis of the immunoblots displays partial inhibition of Akt phosphorylation by MLN3897 in MM.1S and complete inhibition in OPM1 cells. Results are expressed as mean ( \pm SD).

suggesting that OCs induce migration of MM cells mainly by CCL3 secretion (Figure 5B).

Chemokine receptors interact with integrins to enhance cell adhesion by inducing conformational modifications ${ }^{21,27}$; we therefore next assessed whether MLN3897 inhibited adhesion between MM cells and OCs. MM1.S or INA6 cells were plated onto OCs or fibronectin-coated plates for 6 hours in the presence or absence of MLN3897. MM cell adhesion to OCs was almost completely inhibited by MLN3897, independent of CCR1 expression on MM cells (Figure 5C). Importantly, there were no effects on MM cell adhesion to fibronectin (Figure 5C). Although previous reports have shown that integrin was upregulated by CCL3, ${ }^{8,21}$ VLA5 surface expression on MM1.S and INA6 cells was not altered either by CCL3 $(5 \mathrm{ng} / \mathrm{mL}$ for 6 hours)
A
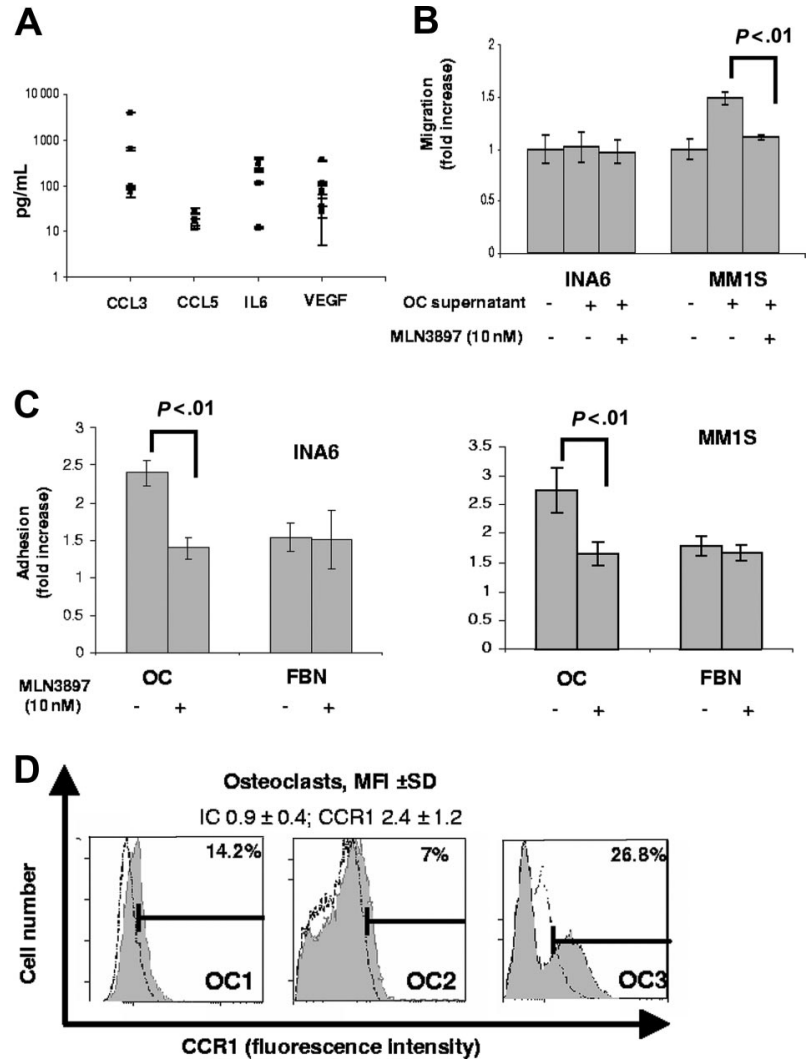

Figure 5. MLN3897 inhibits MM cell migration and adhesion to OCs. (A) CCL3, CCL5, IL-6, and VEGF secretion was assessed by ELISA in OC supernatants at 48 hours; means ( \pm SD) of 3 independent experiments are expressed as $\mathrm{pg} / \mathrm{mL}$ on a logarithmic scale. (B) Effects of MLN3897 on cell migration triggered by 48-hour OC supernatants, assessed by Transwell migration assay. INA6 and MM1.S cell lines were preincubated with MLN3897 (10 nM for 4 hours) and seeded in the upper chamber of the Transwell plate. After 4 hours, cells that migrated to the lower chamber were counted. Results are expressed as fold increase over control. (C) Adhesion assay was performed seeding calcein-labeled INA6 and MM1.S cells on OCs (10 000 cells/well) or fibronectin (FBN; $20 \mu \mathrm{g} / \mathrm{mL}$ ) in the presence or absence of MLN3897. After 6 hours, nonadherent cells were washed off, and fluorescence intensity of adherent cells were expressed as fold increase over control. (D) Representative CCR 1 expression on mature OCs from 3 different donors, analyzed by flow cytometry. Results are expressed as MFI ( \pm SD) of CCR1 and isotype control (IC) from more than 3 independent experiments.

or coculture with OC (Figure S1, available on the Blood website; see the Supplemental Materials link at the top of the online article). Since mature OCs express surface CCR1 $(\mathrm{MFI} \pm \mathrm{SD}, 2.4 \pm 1.2$; Figure 5D), MLN3897 could impair adhesion by direct action on OCs. These data suggest that MLN3897 targets not only MM cells, but also the OC compartment.

\section{MLN3897 abrogates MM cell survival and proliferative advantage conferred by OCs}

It has been shown that OCs enhance MM cell survival, mainly by cell-to-cell contact. ${ }^{2}$ Since MLN3897 almost completely abrogates adhesion of MM cells to OCs, we next examined the growth inhibitory effect of MLN3897 in a long-term OC-MM coculture experiment. OCs were plated in a 24 -well plate $\left(3 \times 10^{4}\right.$ cells/well $)$ with either INA6 MM cells $\left(5 \times 10^{3}\right.$ cells/well $)$ or primary patient MM cells $\left(3 \times 10^{5}\right.$ cells/well $)$ for 5 days. As shown in Figure 6A, OCs promote proliferation and survival of MM cells, consistent with a previous report. ${ }^{2}$ Importantly, MLN3897 almost completely abrogated this survival advantage in both INA6 and primary MM 
A
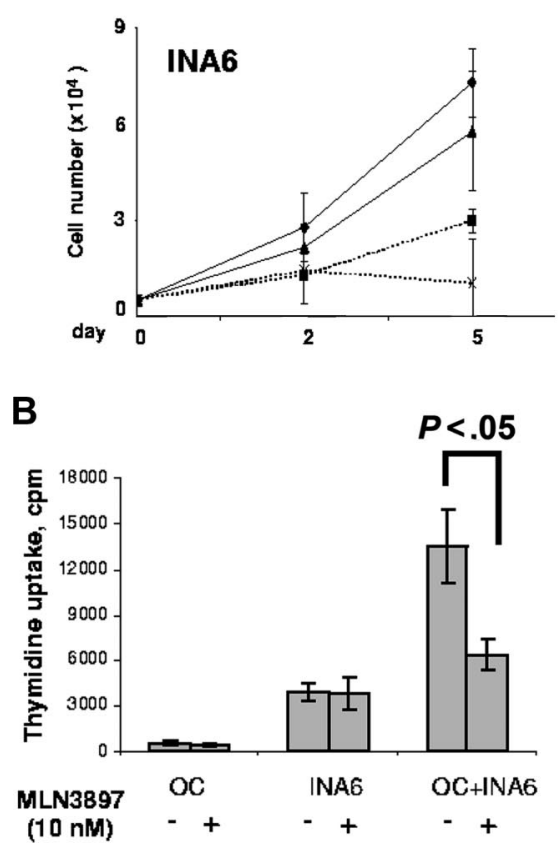
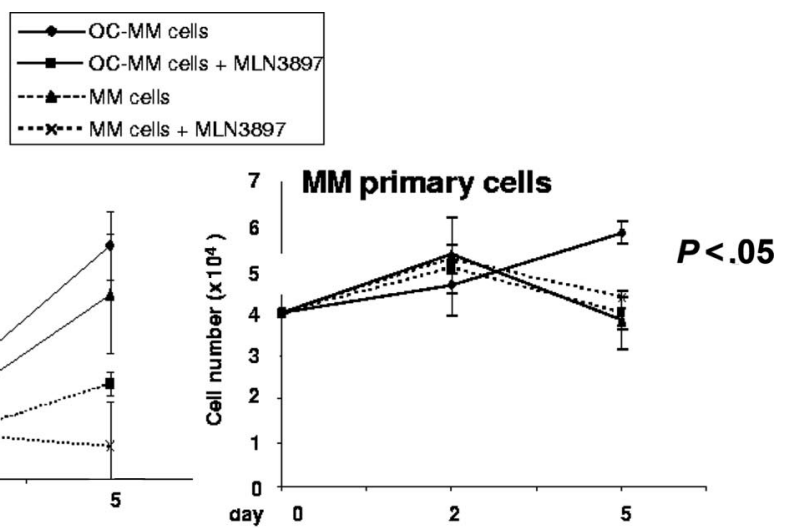

C

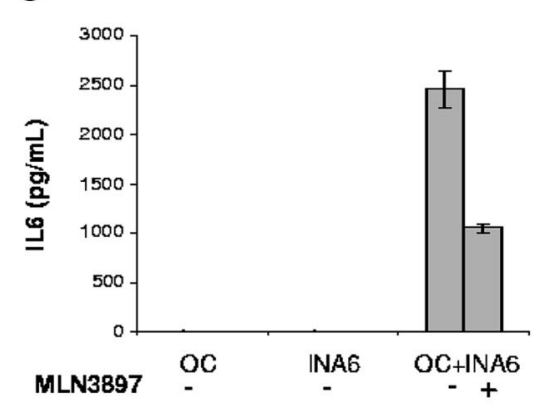

Figure 6. MLN3897 overcomes the survival and proliferation advantage conferred by OCs in coculture. (A) We assessed cell survival of INA6 and MM primary cells alone and in the presence of OCs, with or without MLN3897. At 2 and 5 days, viable cells were counted by trypan blue staining. (B) INA6 cells were cocultured with mature OCs with or without MLN3897 (10 nM) and cell proliferation was assessed by thymidine uptake at 48 hours. Results are expressed as means $( \pm S D)$ counts per minute (cpm). (C) At the same time point, IL-6 secretion was evaluated by ELISA. Results are expressed as mean $( \pm \mathrm{SD})$. cells (Figure 6A). This same effect was also noted in MM1.S cells (data not shown).

We next analyzed whether MLN3897 affected proliferation of INA6 MM cells during short-term culture. OCs were seeded in a 96-well plate at a density of $10^{4}$ cells/well and cocultured with INA6 cells $\left(3 \times 10^{4}\right.$ cells/well $)$ for 48 hours. Our data show that OCs stimulate MM cell proliferation, as assessed at 48 hours by thymidine uptake, in INA6 cells (3.5-fold increase over that of control; Figure 6B) and other cell lines (data not shown). Of note, MLN3897 showed only modest antiproliferative effects on MM cells alone; however, it blocked induction of proliferation by OCs, reducing MM cell proliferative response by 50\% $(P<.05$; Figure 6B). Importantly, CCR1 expression on INA6 cells is lacking (Figure 3A; Table 1), nor is there significant up-regulation of CCR1 expression triggered by coculture with OCs (data not shown). Coculture of INA6 cells significantly enhanced IL-6 secretion from OCs (Figure 6C); conversely, MLN3897 reduced IL-6 secretion by $50 \%(2500 \pm 182 \mathrm{pg} / \mathrm{mL}$ to $1000 \pm 42 \mathrm{pg} / \mathrm{mL})$, suggesting that down-regulation of IL-6 secretion accounts, at least in part, for MLN3897-induced growth inhibition.

\section{Discussion}

OCs within the BM compartment are key regulators in the pathogenesis of MM bone disease. OCs and MM cells interact to promote the others' survival and proliferation, thereby contributing to both OBD and drug resistance. MM cells enhance OC formation and bone resorption by secretion of osteoclastogenic cytokines such as RANKL, VEGF, CCL3, and IL-6.9,22,28-30 In turn, OCs sustain tumor cell survival and protect malignant plasma cells from chemotherapy-induced apoptosis, ${ }^{2}$ mainly by cell-to-cell contact and cytokine secretion. The development of OBD depends on this loop between OCs and MM cells, and the involvement of chemokines such as CCL3 is critical. The role of CCL3 is complex; not only is it secreted by both OCs and MM cells, but it also interacts with 2 receptors, CCR 1 and CCR5, expressed by both cell types. CCR1 is the most frequent chemokine receptor on OCs, ${ }^{31}$ mediating CCL3-induced OC formation and function. ${ }^{11,21}$ Furthermore, CCL3 stimulates migration, adhesion, and survival of MM cells via CCR1 and CCR5, although the role of each receptor in mediating these effects remains to be elucidated. ${ }^{6,10,21,24}$ Here, we studied the in vitro effects and molecular mechanisms of action of MLN3897, a novel oral small-molecule CCR1 inhibitor currently undergoing phase 2 clinical testing in multiple sclerosis and rheumatoid arthritis. MLN3897 impairs OC differentiation by blocking the fusion process and by down-regulating ERK and c-fos signaling. Importantly, MLN3897 specifically affects OC-MM cell interactions by abrogation of CCL3 and OC-induced migration of MM cells, as well as by inhibiting OCs to MM cell adhesion. Finally, we show that MLN3897 abrogates the survival and proliferative advantage conferred by OCs to MM cell lines and primary MM cells. Thus, MLN3897 provides a novel strategy to efficiently target $\mathrm{OBD}$ in MM.

During osteoclastogenesis, precursor cells secrete chemokines such as CCL3, CCL5, and CCL2, thereby enhancing migration of OC precursor cells and promoting fusion. ${ }^{16,32}$ The main receptor for CCL3 on OCs is CCR $1^{11,31}$; conversely, CCR 1 inhibition significantly reduces the number of OCs in an in vivo model. ${ }^{21,24}$ Consistent with these data, MLN3897 effectively blocked human PBMC-derived OC formation and OC activity, mainly by inhibiting fusion of precursor cells. The multinucleation step in OC differentiation is regulated by c-fos. ${ }^{20,33} \mathrm{In}$ particular, osteoclastogenesis depends on stable c-fos expression maintained by sustained ERK signaling. ${ }^{34}$ Here, we show that treatment with MLN3897 down-regulates pERK and c-fos 
expression, which is associated with inhibition of multinucleated cell formation. Whether these 2 effects are casually related remains to be determined. Interestingly, MLN3897 does not completely suppress OC number and activity, suggesting that other chemokine receptors may partially compensate for the effects of CCR1 inhibition of OCs.

As previously reported, ${ }^{6,23}$ almost all MM cell lines and primary cells secrete CCL3, which is an important mediator of MM effects on OCs. CCL3 in turn affects MM cells by activation of both CCR1 and CCR5. At present, reports on expression of these receptors on MM cells are controversial ${ }^{6,7,10,21}$ : Lentzsch et al ${ }^{6}$ reported surface CCR5 expression on the MM1.S cell line, whereas Oba et $\mathrm{al}^{21}$ could not identify this receptor at the RNA level. In our series, surface CCR1 expression on MM cell lines was only modest; 2 cell lines (MM1.S and OPM1) displayed significant expression levels, while low levels were observed on primary MM cells. Interestingly, CCL3-induced migration of MM cells was correlated with CCR1 expression and activation of Akt; conversely, treatment with MLN3897 inhibited CCL3-induced migration and Akt activation in MM1.S and OPM1 cells. Since MM1.S cells express both CCL3 receptors, these data indicate that CCR5, in addition to CCR1, may mediate CCL3 effects, as suggested by prior reports. ${ }^{24}$ Moreover, MLN3897 partially inhibited CCL3 effects on MM1.S cell migration and signaling, suggesting stronger inhibition of CCR1, although effects on CCR5 cannot be excluded.

OCs interact with cancer cells in multiple ways. For example, OCs secrete cytokines, thereby inducing homing of MM cells to $\mathrm{BM}^{24}$ in $\mathrm{MM}$ and promoting bone metastasis in other solid tumors like lung cancer. ${ }^{35}$ Here, we show that OCs promote CCR $1^{+} \mathrm{MM}$ cell migration by secretion of several chemokines, mainly CCL3, and that pretreatment with MLN3897 efficiently inhibits this effect. Importantly, we cannot exclude involvement of other chemokines stimulating MM cell migration and proliferation. Indeed, more than 11 chemokines have been identified as CCR1 ligands ${ }^{4}$; of these, CCL3 and CCL7 are secreted by OCs and mediate MM cell homing to the BM. ${ }^{11,36}$ Chemokines and their receptors also enhance cell adhesion by interaction with integrins by modifying surface expression, lateral mobility, or binding sites. ${ }^{27,37,38}$ MLN3897 inhibits MM cell-OC cell contact by inhibition of adhesion regardless of CCR1 expression on tumor cells, suggesting that CCR1 inhibition mainly targets the OC compartment. Both cytokine secretion and cell-to-cell adhesion are critical mediators of the survival effects of OCs conferred on MM cells. ${ }^{2}$ Treatment with MLN3897 abrogates not only the survival advantage conferred by OCs to MM cells, but also the enhanced proliferation, due to both reduced adhesion of MM cells to OCs and impaired cytokine secretion. In contrast to previous studies, ${ }^{8,21}$ we did not observe significant up-regulation of VLA5 surface expression on MM cells triggered either by stimulation with CCL3 or coculture with OCs, likely due to the shorter time points we analyzed. Ongoing studies are addressing the mechanism for the specific inhibitory effect of MLN3897 on MM cell-to-OC adhesion.

Our data therefore demonstrate that CCR1 inhibition by MLN3897 may be an effective tool to block and possibly even prevent OBD in MM. MLN3897 blocks OC development and function by inhibiting differentiation of OC precursors, in particular by down-regulating cell fusion and c-fos expression. Moreover, MLN3897 abrogates MM cell migration and adhesion to OCs, thus preventing the homing of MM cells to OC sites. Finally, MLN3897 overcomes the protective effect of OCs on MM cell survival and proliferation, thereby inhibiting the interactive loop between OCs and MM cells. Ongoing studies are defining the in vivo effects of MLN3897 in a severe combined immunodeficiency-human (SCIDhu) mouse model of MM-related osteolysis. ${ }^{39}$ These data therefore delineate a novel mechanism of action of MLN3897 on osteoclastogenesis and $\mathrm{OC}-\mathrm{MM}$ cell interactions, and provide the preclinical rationale for its clinical evaluation to treat $\mathrm{OBD}$ in $\mathrm{MM}$.

\section{Acknowledgments}

We thank Zhenxin Shen and Petter Veiby for their valuable contributions and assistance in preparing this manuscript.

This work was supported by Multiple Myeloma Research Foundation Senior Award (N.R.); NIH Grant Specialized Programs of Research Excellence (SPORE) IP50 CA10070-01, PO-1 78378, AND RO-1 CA 50947; and the Doris Duke Distinguished Clinical Research Scientific Award (K.C.A.). S.V. is supported by a fellowship from Millennium Pharmaceuticals.

\section{Authorship}

S.V. designed research, performed research, analyzed data, and wrote the paper; N.R., T.H., and K.C.A. designed research and wrote the paper; K.I. and I.B. performed research and analyzed data; K.P., T.K., and H.Y. performed and designed research; S.C., S.P., N.S., S.M., and N.V. performed research; E.M.O., J.J., Y.O., H.I., and D.C. analyzed data; and M.L. and M.B. designed research.

Conflict-of-interest disclosure: The authors declare no competing financial interests.

Correspondence: Kenneth C. Anderson, Dana-Farber Cancer Institute, Mayer 557, 44 Binney St, Boston, MA 02115; e-mail: kenneth_anderson@dfci.harvard.edu.

\section{References}

1. Body JJ. Effectiveness and cost of bisphosphonate therapy in tumor bone disease. Cancer. 2003;97:859-865

2. Abe M, Hiura K, Wilde J, et al. Osteoclasts enhance myeloma cell growth and survival via cell-cell contact: a vicious cycle between bone destruction and myeloma expansion. Blood. 2004;104:2484-2491.

3. Charo IF, Ransohoff RM. The many roles of chemokines and chemokine receptors in inflammation. N Engl J Med. 2006;354:610-621.

4. Tian Y, New DC, Yung LY, et al. Differential chemokine activation of CC chemokine receptor 1-regulated pathways: ligand selective activation of Galpha 14-coupled pathways. Eur J Immunol. 2004;34:785-795.

5. Godessart N. Chemokine receptors: attractive targets for drug discovery. Ann N Y Acad Sci. 2005; 1051:647-657.

6. Lentzsch S, Gries M, Janz M, Bargou R, Dorken $B$, Mapara MY. Macrophage inflammatory protein 1-alpha (MIP-1 alpha) triggers migration and signaling cascades mediating survival and proliferation in multiple myeloma (MM) cells. Blood. 2003; 101:3568-3573.

7. Han JH, Choi SJ, Kurihara N, Koide M, Oba Y, Roodman GD. Macrophage inflammatory protein1alpha is an osteoclastogenic factor in myeloma that is independent of receptor activator of nuclear factor kappaB ligand. Blood. 2001;97: 3349-3353.

8. Choi SJ, Oba Y, Gazitt Y, et al. Antisense inhibition of macrophage inflammatory protein 1-alpha blocks bone destruction in a model of myeloma bone disease. J Clin Invest. 2001;108:18331841.

9. Oyajobi BO, Franchin G, Williams PJ, et al. Dual effects of macrophage inflammatory protein-1alpha on osteolysis and tumor burden in the murine 5TGM1 model of myeloma bone disease. Blood. 2003;102:311-319.

10. Moller C, Stromberg T, Juremalm M, Nilsson K, Nilsson $\mathrm{G}$. Expression and function of chemokine receptors in human multiple myeloma. Leukemia. 2003;17:203-210.

11. $Y u X$, Huang $Y$, Collin-Osdoby $P$, Osdoby $P$. CCR1 chemokines promote the chemotactic recruitment, RANKL development, and motility of 
osteoclasts and are induced by inflammatory cytokines in osteoblasts. J Bone Miner Res. 2004; 19:2065-2077.

12. Ishida N, Hayashi K, Hattori A, Yogo K, Kimura T, Takeya T. CCR1 acts downstream of NFAT2 in osteoclastogenesis and enhances cell migration. J Bone Miner Res. 2006;21:48-57.

13. Burger R, Guenther A, Bakker F, et al. Gp130 and ras mediated signaling in human plasma cell line INA-6: a cytokine-regulated tumor model for plasmacytoma. Hematol J. 2001;2:42-53.

14. Kiziltepe T, Hideshima T, Ishitsuka K, et al. JS-K, a GST-activated nitric oxide generator, induces DNA double-strand breaks, activates DNA damage response pathways, and induces apoptosis in vitro and in vivo in human multiple myeloma cells. Blood. 2007;110:709-718.

15. Rasband WS. ImageJ. Bethesda, MD: U.S. National Institutes of Health. 1997-2006. Available at: http://rsb.info.nih.gov/ij/. Accessed September 13, 2007.

16. Kim MS, Day CJ, Selinger $\mathrm{Cl}$, Magno CL, Stephens SR, Morrison NA. MCP-1-induced human osteoclast-like cells are tartrate-resistant acid phosphatase, NFATc1, and calcitonin receptorpositive but require receptor activator of NFkappaB ligand for bone resorption. J Biol Chem. 2006;281:1274-1285.

17. Hotokezaka H, Sakai E, Ohara N, et al. Molecular analysis of RANKL-independent cell fusion of osteoclast-like cells induced by TNF-alpha, lipopolysaccharide, or peptidoglycan. J Cell Biochem. 2007;101:122-134.

18. Murphy LO, Smith S, Chen RH, Fingar DC, Blenis J. Molecular interpretation of ERK signal duration by immediate early gene products. Nat Cell Biol. 2002;4:556-564.

19. Faccio R, Takeshita S, Zallone A, Ross FP, Teitelbaum SL. C-Fms and the alphavbeta3 integrin collaborate during osteoclast differentiation. J Clin Invest. 2003;111:749-758.

20. Ishida N, Hayashi K, Hoshijima M, et al. Large scale gene expression analysis of osteoclastogenesis in vitro and elucidation of NFAT2 as a key regulator. J Biol Chem. 2002;277:41147-41156.
21. Oba Y, Lee JW, Ehrlich LA, et al. MIP-1alpha utilizes both CCR1 and CCR5 to induce osteoclast formation and increase adhesion of myeloma cells to marrow stromal cells. Exp Hematol. 2005; 33:272-278.

22. Abe M, Hiura K, Wilde J, et al. Role for macrophage inflammatory protein (MIP) $-1 \alpha$ and MIP-1 $\beta$ in the development of osteolytic lesions in multiple myeloma. Blood. 2002;100:2195-2202.

23. Choi SJ, Cruz JC, Craig F, et al. Macrophage inflammatory protein 1-alpha is a potential osteoclast stimulatory factor in multiple myeloma. Blood. 2000;96:671-675.

24. Menu E, De Leenheer E, De Raeve H, et al. Role of CCR 1 and CCR5 in homing and growth of multiple myeloma and in the development of osteolytic lesions: a study in the 5TMM model. Clin Exp Metastasis. 2006;23:291-300.

25. Wang J, Zhang X, Thomas SM, et al. Chemokine receptor 7 activates phosphoinositide-3 kinasemediated invasive and prosurvival pathways in head and neck cancer cells independent of EGFR. Oncogene. 2005;24:5897-5904.

26. Tombran-Tink J, Barnstable CJ. Osteoblasts and osteoclasts express PEDF, VEGF-A isoforms, and VEGF receptors: possible mediators of angiogenesis and matrix remodeling in the bone. Biochem Biophys Res Commun. 2004;316:573579 .

27. Weber C, Kitayama J, Springer TA. Differential regulation of beta 1 and beta 2 integrin avidity by chemoattractants in eosinophils. Proc Natl Acad Sci U S A. 1996;93:10939-10944.

28. Aldridge SE, Lennard TW, Williams JR, Birch MA Vascular endothelial growth factor acts as an osteolytic factor in breast cancer metastases to bone. Br J Cancer. 2005;92:1531-1537.

29. Sezer O, Heider U, Jakob C, et al. Immunocytochemistry reveals RANKL expression of myeloma cells. Blood. 2002;99:4646-4647.

30. Barille S, Collette M, Bataille R, Amiot M. Myeloma cells upregulate interleukin- 6 secretion in osteoblastic cells through cell-to-cell contact but downregulate osteocalcin. Blood. 1995;86:31513159 .
31. Lean JM, Murphy C, Fuller K, Chambers TJ. CCL9/MIP-1gamma and its receptor CCR1 are the major chemokine ligand/receptor species expressed by osteoclasts. J Cell Biochem. 2002;87: 386-393.

32. Kim MS, Magno CL, Day CJ, Morrison NA. Induction of chemokines and chemokine receptors CCR2b and CCR4 in authentic human osteoclasts differentiated with RANKL and osteoclast like cells differentiated by MCP-1 and RANTES. J Cell Biochem. 2006;97:512-518.

33. Grigoriadis AE, Wang ZQ, Cecchini MG, et al. c-Fos: a key regulator of osteoclast-macrophage lineage determination and bone remodeling. Science. 1994;266:443-448.

34. Coronella-Wood J, Terrand J, Sun H, Chen QM. c-Fos phosphorylation induced by $\mathrm{H} 2 \mathrm{O} 2$ prevents proteasomal degradation of c-Fos in cardiomyocytes. J Biol Chem. 2004;279:33567-33574.

35. Nakamura ES, Koizumi K, Kobayashi M, et al. RANKL-induced CCL22/macrophage-derived chemokine produced from osteoclasts potentially promotes the bone metastasis of lung cancer expressing its receptor CCR4. Clin Exp Metastasis. 2006;23:9-18

36. Vande Broek I, Asosingh K, Vanderkerken K, Straetmans N, Van Camp B, Van Riet I. Chemokine receptor CCR2 is expressed by human multiple myeloma cells and mediates migration to bone marrow stromal cell-produced monocyte chemotactic proteins MCP-1, -2 and -3 . Br J Cancer. 2003;88:855-862.

37. Weber C, Alon R, Moser B, Springer TA. Sequential regulation of alpha 4 beta 1 and alpha 5 beta 1 integrin avidity by CC chemokines in monocytes: implications for transendothelial chemotaxis. J Cell Biol. 1996;134:1063-1073.

38. Grabovsky V, Feigelson S, Chen C, et al. Subsecond induction of alpha4 integrin clustering by immobilized chemokines stimulates leukocyte tethering and rolling on endothelial vascular cell adhesion molecule 1 under flow conditions. J Exp Med. 2000;192:495-506.

39. Tassone P, Neri P, Carrasco DR, et al. A clinically relevant SCID-hu in vivo model of human multiple myeloma. Blood. 2005;106:713-716. 